\title{
GENERALIZATIONS OF THE SPHERICAL MEAN CONVEXITY THEOREM ON SUBHARMONIC FUNCTIONS
}

\author{
N.A. Watson \\ University of Canterbury, Department of Mathematics \\ Christchurch 1, New Zealand; naw@math.canterbury.ac.nz
}

\begin{abstract}
An apparently new proof of the theorem in the title is given. The theorem is then generalized to mean values over level surfaces of Green functions, of subharmonic functions associated with a self-adjoint elliptic partial differential operator with variable coefficients. The analogue for subtemperatures is also proved by a similar method.
\end{abstract}

\section{Introduction}

Let $u$ be subharmonic on an annulus $\left\{x \in \mathbf{R}^{n}: r_{1}<\left\|x_{0}-x\right\|<r_{2}\right\}$, and let $\tau(r)=-\log r$ if $n=2, \tau(r)=r^{2-n}$ if $n \geq 3$. A classical result of F. Riesz states that the integral mean of $u$ over $\left\{x:\left\|x_{0}-x\right\|=r\right\}$ is a convex function of $\tau(r)$ for $r \in] r_{1}, r_{2}$ [. We give below a simple, apparently new proof of this result, that uses only Green's formula and a standard approximation argument. The proof invites generalization, and we proceed to give analogues for subsolutions of both second order, linear, elliptic partial differential equations with variable coefficients, and the heat equation. The general elliptic case is far from elementary, since the necessary existence and properties of the fundamental solution, the monotone approximation of arbitrary subsolutions by smooth ones, and even the existence of suitable surfaces in sufficient quantity, all require sophisticated theorems. For parabolic partial differential equations, there is apparently no suitable approximation theorem in the case of variable coefficients, so we restrict our attention to the heat equation, in which context the method was first discovered.

For clarity of exposition, we give the new proof of Riesz's theorem first. In the general elliptic case, the means may be defined only almost everywhere, so we next show that if a function is defined and satisfies the usual convexity inequality almost everywhere on an interval in $\mathbf{R}$, then it can be extended to a function convex on the whole interval. We then consider the general elliptic case in our main result (Theorem 2), and incorporate recent generalizations of Riesz's theorem to means over level surfaces of Green functions, proved for the Laplacian case in [19]. We subsequently extend these results to means of $L^{p}$ and similar type, using the elementary technique of Fugard [4], which fits in very well with our overall

1991 Mathematics Subject Classification: Primary 31B35; Secondary 35B05, 35J15, 35K05.

doi:10.5186/aasfm.1992.1733 
approach. Finally, we consider the case of the heat equation, where the only real difference is that the singularity of the Green function must be handled in a different way.

It is a pleasure to thank David Armitage, Heinz Bauer and Joel Schiff for their help in obtaining material essential to the general elliptic case, and the referee for helpful and informative comments.

\section{The proof in the classical case}

On any smooth surface, we use $\sigma$ to denote surface area measure, and $D_{\nu}$ to denote differentiation along the outward normal. Let $G\left(x_{0}, \cdot\right)$ denote the fundamental superharmonic function with pole at $x_{0} \in \mathbf{R}^{n}$, let $A\left(x_{0}, r_{1}, r_{2}\right)$ denote $\left\{x \in \mathbf{R}^{n}: r_{1}<\left\|x_{0}-x\right\|<r_{2}\right\}$, let $B\left(x_{0}, r\right)=\left\{x \in \mathbf{R}^{n}:\left\|x_{0}-x\right\|<r\right\}$, and put

$$
\mathscr{L}\left(u, x_{0}, r\right)=-\int_{\partial B\left(x_{0}, r\right)} u D_{\nu} G\left(x_{0}, \cdot\right) d \sigma .
$$

Then $\mathscr{L}\left(u, x_{0}, r\right)$ differs from the usual spherical average of $u$ by a positive constant multiple, which does not affect our results.

Theorem 1 (F. Riesz [13]). If $u$ is subharmonic on $A\left(x_{0}, r_{1}, r_{2}\right)$, then there is a convex function $\varphi$ such that $\mathscr{L}\left(u, x_{0}, \cdot\right)=\varphi \circ \tau$ on $] r_{1}, r_{2}[$.

Proof. Suppose first that $u \in C^{2}$. Let $r_{1}<s_{1}<s_{2}<r_{2}$, and put $A=$ $A\left(x_{0}, s_{1}, s_{2}\right)$. If $v$ is harmonic on $A\left(x_{0}, r_{1}, r_{2}\right)$, it follows from Green's formula that

$$
\int_{A} v \Delta u d x=\int_{\partial A}\left(v D_{\nu} u-u D_{\nu} v\right) d \sigma
$$

Taking $v=1$, we obtain

$$
\int_{A} \Delta u d x=\int_{\partial A} D_{\nu} u d \sigma
$$

If we put $v=G=G\left(x_{0}, \cdot\right)$ in (1), we obtain

$$
\begin{aligned}
\int_{A} G \Delta u d x= & \tau\left(s_{2}\right) \int_{\partial B\left(s_{2}\right)} D_{\nu} u d \sigma-\tau\left(s_{1}\right) \int_{\partial B\left(s_{1}\right)} D_{\nu} u d \sigma \\
& \quad-\int_{\partial B\left(s_{2}\right)} u D_{\nu} G d \sigma+\int_{\partial B\left(s_{1}\right)} u D_{\nu} G d \sigma \\
= & \tau\left(s_{2}\right) \kappa\left(s_{2}\right)-\tau\left(s_{1}\right) \kappa\left(s_{1}\right)+\mathscr{L}\left(s_{2}\right)-\mathscr{L}\left(s_{1}\right),
\end{aligned}
$$


where $B(s)=B\left(x_{0}, s\right), \mathscr{L}(s)=\mathscr{L}\left(u, x_{0}, s\right)$, and

$$
\kappa(s)=\int_{\partial B(s)} D_{\nu} u d \sigma
$$

Note that, by (2),

$$
\kappa\left(s_{2}\right)-\kappa\left(s_{1}\right)=\int_{A} \Delta u d x
$$

It follows that

$$
\begin{aligned}
\frac{\mathscr{L}\left(s_{2}\right)-\mathscr{L}\left(s_{1}\right)}{\tau\left(s_{2}\right)-\tau\left(s_{1}\right)} & =\frac{1}{\tau\left(s_{2}\right)-\tau\left(s_{1}\right)}\left(\int_{A} G \Delta u d x-\tau\left(s_{1}\right)\left(\kappa\left(s_{2}\right)-\kappa\left(s_{1}\right)\right)\right)-\kappa\left(s_{2}\right) \\
& =\int_{A}\left(\frac{G-\tau\left(s_{1}\right)}{\tau\left(s_{2}\right)-\tau\left(s_{1}\right)}\right) \Delta u d x-\kappa\left(s_{2}\right) .
\end{aligned}
$$

Therefore, if $r_{1}<r<s<t<r_{2}$ and $A(p, q)=A\left(x_{0}, p, q\right)$, we have

$$
\begin{aligned}
& \frac{\mathscr{L}(t)-\mathscr{L}(s)}{\tau(t)-\tau(s)}-\frac{\mathscr{L}(s)-\mathscr{L}(r)}{\tau(s)-\tau(r)} \\
& \quad=\int_{A(s, t)}\left(\frac{G-\tau(s)}{\tau(t)-\tau(s)}\right) \Delta u d x-\kappa(t)-\int_{A(r, s)}\left(\frac{G-\tau(r)}{\tau(s)-\tau(r)}\right) \Delta u d x+\kappa(s) \\
& =\int_{A(s, t)}\left(\frac{G-\tau(t)}{\tau(t)-\tau(s)}\right) \Delta u d x+\int_{A(r, s)}\left(\frac{\tau(r)-G}{\tau(s)-\tau(r)}\right) \Delta u d x,
\end{aligned}
$$

in view of (3). Since $\tau(s)>G>\tau(t)$ on $A(s, t)$, and $\tau(r)>G>\tau(s)$ on $A(r, s)$, the last two integrals are both non-positive. This proves the reuslt for smooth subharmonic functions.

Suppose now that $u$ is arbitrary. Let $r_{1}<s_{1}<s_{2}<r_{2}$, and take a decreasing sequence $\left\{u_{j}\right\}$ of smooth subharmonic functions which converges to $u$ on a neighbourhood of $\bar{A}\left(s_{1}, s_{2}\right)$. Then, for each $j$, there is a convex function $\varphi_{j}$ such that $\mathscr{L}\left(u_{j}, x_{0}, \cdot\right)=\varphi_{j} \circ \tau$ on $] s_{1}, s_{2}[$, so that

$$
\mathscr{L}\left(u, x_{0}, \cdot\right)=\lim \mathscr{L}\left(u_{j}, x_{0}, \cdot\right)=\lim \varphi_{j} \circ \tau,
$$

and $\lim \varphi_{j}$ is convex because $\mathscr{L}\left(u, x_{0}, \cdot\right)$ is finite. Since $s_{1}, s_{2}$ are arbitrary, this completes the proof.

There are, of course, several known proofs of Theorem 1. The method of Riesz [13] involves the Dirichlet problem; those of Doob [3, p. 24] and Gardiner [5] rely on symmetry properties of the sphere; and that of Dinghas [2] is elementary but complicated. Another proof, supplied by the referee, is as elementary and less complicated than the one given above; but it does not generalize easily. Thus none of the earlier proofs are so inviting of generalization. 


\section{Convexity of functions defined on a dense subset of an interval}

In this section, we show that a function which is defined and satisfies the usual convexity inequality on a dense subset of an open interval in $\mathbf{R}$, can be extended to a function convex on the whole interval.

Definition. Let $J$ be a dense subset of a bounded open interval $I \subseteq \mathbf{R}$. A function $f: J \rightarrow \mathbf{R}$ is called convex if

$$
f((1-t) a+t b) \leq(1-t) f(a)+t f(b)
$$

whenever $0 \leq t \leq 1$ and $a, b,(1-t) a+t b \in J$.

We derive some basic properties of $f$, following the treatment of functions convex on $I$ in [15, p. 199]. Thus, if $a, b, c \in J$ and $a<b<c$, then

$$
\frac{f(b)-f(a)}{b-a} \leq \frac{f(c)-f(a)}{c-a} \leq \frac{f(c)-f(b)}{c-b} .
$$

Given any $x \in J$, define a function $\psi$ on the set $J_{x}=\{h \neq 0: x+h \in J\}$ by

$$
\psi(h)=\frac{f(x+h)-f(x)}{h} .
$$

Then (4) shows that $\psi$ is increasing (in the wide sense) on $J_{x}$, so that, taking limits through the set $J_{x}$, we have $\psi(0-) \leq \psi(0+)$. Defining $f_{+}^{\prime}(x)$ to be $\psi(0+)$, and $f_{-}^{\prime}(x)$ to be $\psi\left(0_{-}\right)$, we obtain functions $f_{+}^{\prime}, f_{-}^{\prime}: J \rightarrow \mathbf{R}$ such that $f_{-}^{\prime} \leq f_{+}^{\prime}$. These functions are increasing on $J$; for if $a, b, c \in J$ and $a<b$, it follows from (4) that

$$
f_{+}^{\prime}(a) \leq \frac{f(b)-f(a)}{b-a} \leq \lim _{c \rightarrow b+} \frac{f(c)-f(b)}{c-b}=f_{+}^{\prime}(b),
$$

and similarly that $f_{-}^{\prime}(a) \leq f_{-}^{\prime}(b)$. We can now deduce that $f$ is locally uniformly continuous on $J$. For if $\alpha, \beta \in J$ and $M=\max \left\{\left|f_{+}^{\prime}(\alpha)\right|,\left|f_{-}^{\prime}(\beta)\right|\right\}$, then whenever $x, y \in J$ and $\alpha \leq x<y \leq \beta$ we have

$$
-M \leq f_{+}^{\prime}(\alpha) \leq f_{+}^{\prime}(x) \leq \frac{f(x)-f(y)}{x-y} \leq f_{-}^{\prime}(y) \leq f_{-}^{\prime}(\beta) \leq M,
$$

so that $|f(x)-f(y)| \leq M|x-y|$. It now follows [7, p. 169] that $f$ can be extended to a locally uniformly continuous function on $I$, and the convexity of the extension then follows, by continuity, from that of $f$. 


\section{Elliptic equations with variable coefficients}

We consider a self-adjoint, uniformly elliptic operator

$$
L u=\sum_{i, j=1}^{n} D_{i}\left(a_{i j} D_{j} u\right)
$$

where each $a_{i j}$ and its first and second order partial derivatives are assumed to be bounded and locally Lipschitz continuous on $\mathbf{R}^{n}$, and $a_{i j}=a_{j i}$.

If $E$ is a subdomain of $\mathbf{R}^{n}$, then a locally integrable function $u$ on $E$ is said to satisfy $L u \geq 0$ weakly if

$$
\int_{E} u L v d x \geq 0
$$

whenever $v \geq 0$ and $v \in C_{c}^{2}(E)$. Such functions were studied by Littman [10], who showed that each is equal a.e. to an upper semicontinuous function that is locally the pointwise limit of a decreasing sequence of $C^{2}$ functions $\left\{u_{j}\right\}$ such that $L u_{j} \geq 0$. Thus $u$ can be identified with a function $L$-subharmonic in the sense of Hervé [8]. We shall adopt Hervé's terminology for all potential theoretic notions relative to $L$. Details of these can all be found in [8] or [1].

Throughout this section, we denote by $D$ a subdomain of $\mathbf{R}^{n}$ that possesses a Green function in the following (classical) sense. Given any $z \in D$, there is a positive function $G_{D}(z, \cdot)$ on $D$ such that:

(i) $L G_{D}(z, \cdot)=0$ on $D \backslash\{z\} ; G_{D}(z, z)=\infty$;

(ii) $G_{D}(z, \cdot)$ can be continuously extended to zero on $\partial D$, including the point at infinity if $D$ is unbounded;

(iii) as $y \rightarrow z$,

$$
G_{D}(z, y) \sim\left(\sum_{i, j=1}^{n} b_{i j}(z)\left(y_{i}-z_{i}\right)\left(y_{j}-z_{j}\right)\right)^{(2-n) / 2}
$$

where $\left(b_{i j}\right)$ is the inverse matrix of $\left(a_{i j}\right)$;

(iv) given any compact subset $K$ of $D$, there are positive constants $c_{1}, c_{2}$ such that

$$
c_{1} \tau(\|z-y\|) \leq G_{D}(z, y) \leq c_{2} \tau(\|z-y\|)
$$

whenever $y, z \in K$.

It is known [8] that (i), (iii) and (iv) hold for any $D$ that supports a positive $L$-potential, where $G_{D}(z, \cdot)$ is an $L$-potential with support $\{z\}$. It follows that, if $E$ is any bounded, Dirichlet regular domain with closure in such a $D$, then (i)-(iv) hold with $E$ in place of $D$, and with $G_{E}(z, \cdot)$ equal to $G_{D}(z, \cdot)$ minus the Dirichlet $L$-solution on $E$ with boundary function $G_{D}(z, \cdot)$. If $n \geq 3$, the 
inequalities in (iv) hold for all $y, z \in \mathbf{R}^{n}$ by [11], so that (i)-(iv) hold also for unbounded Dirichlet regular domains.

We now show that any $L$-subharmonic function $u$ satisfies $L u \geq 0$ weakly. It is sufficient to prove this locally, and so we can assume that $u$ is defined on $D$. If $U$ is an open set with closure in $D$, then $u=h-p$ for some $L$-harmonic function $h$ on $U$, and $L$-potential $p$ on $D$, by a result of Brelot and Hervé [1, p. 97]. Since the $L$-potentials on $D$ with point support are proportional [8, p. 563], there is a non-negative Radon measure $\mu$ on $D$ such that

$$
p(x)=\int_{D} G_{D}(x, y) d \mu(y)
$$

for all $x \in D$ [8, p. 481]. The logarithmic or Newtonian potential of the restriction $\bar{\mu}$ of $\mu$ to $\bar{U}$ is locally integrable, so that (iv) implies that the $L$-potential of $\bar{\mu}$ on $D$ is also locally integrable, from which it follows that $p$ is also. Therefore, if $v \geq 0$ and $v \in C_{c}^{2}(D)$, we have

$$
\int_{D} p(x) L v(x) d x=\int_{D} d \mu(y) \int_{D} G_{D}(x, y) L v(x) d x \leq 0,
$$

since $L G_{D}(\cdot, y) \leq 0$ weakly, so that $L u \geq 0$ weakly.

We now describe the surfaces and means which generalize those in Theorem 1.

For any $x_{0} \in D$ and $r>0$ such that $\tau(r)>0$, the set

$$
B_{D}\left(x_{0}, r\right)=\left\{x \in D: G_{D}\left(x_{0}, x\right)>\tau(r)\right\}
$$

is open and has its closure in $D$ (by (ii)). It is also connected, because if it had a component $C$ that did not contain $x_{0}$, we would have $G_{D}\left(x_{0}, \cdot\right)=\tau(r)$ on $\partial C$ and $L G_{D}\left(x_{0}, \cdot\right)=0$ throughout $C$, which would yield the contradiction $G_{D}\left(x_{0}, \cdot\right)=\tau(r)$ on $C$.

Since $G_{D}\left(x_{0}, \cdot\right) \in C^{2}\left(D \backslash\left\{x_{0}\right\}\right)$, for almost every $r$ such that $\tau(r)>0$ the set

$$
\left\{x \in D: G_{D}\left(x_{0}, x\right)=\tau(r)\right\}
$$

is a $C^{2}$, regular, $(n-1)$-dimensional manifold, by Sard's theorem [14, p. 45]. We call such a value of $r$ a regular value. Whenever $r$ is a regular value, the set (5) is $\partial B_{D}\left(x_{0}, r\right)$, and its outward unit normal is given by the standard formula $\nu=-\nabla G_{D}\left(x_{0}, \cdot\right)\left\|\nabla G_{D}\left(x_{0}, \cdot\right)\right\|^{-1}$.

We shall consider domains bounded by two surfaces of the form (5), where $x_{0}$ is fixed and $r$ assumes two regular values. If $L=\Delta$ and $D=\mathbf{R}^{n}$, then such a domain is an annulus. Given $x_{0} \in D$ and regular values $r_{1}<r_{2}$, we put

$$
A_{D}\left(x_{0}, r_{1}, r_{2}\right)=B_{D}\left(x_{0}, r_{2}\right) \backslash \bar{B}_{D}\left(x_{0}, r_{1}\right) .
$$


The surface means are defined as follows. Given $x_{0} \in D$ and a regular value of $r$, we put

$$
\mathscr{L}_{D}\left(u, x_{0}, r\right)=-\int_{\partial B_{D}\left(x_{0}, r\right)}\left\langle A \nabla G_{D}\left(x_{0}, \cdot\right), \nu\right\rangle u d \sigma
$$

whenever the integral exists, where

$$
\langle A \nabla f, \nu\rangle=\sum_{i, j=1}^{n} a_{i j}\left(D_{j} f\right) \nu_{i}
$$

Such means have been studied independently in [9], where conditions under which they characterize solutions of various elliptic equations are given.

Theorem 2. If $u$ is $L$-subharmonic on $A_{D}\left(x_{0}, r_{1}, r_{2}\right)$, then there is a convex function $\varphi$ on $] \tau\left(r_{2}\right), \tau\left(r_{1}\right)[$ such that

$$
\mathscr{L}_{D}\left(u, x_{0}, r\right)=\varphi(\tau(r))
$$

for all regular values of $r$ in $] r_{1}, r_{2}[$. Moreover, given any $\eta \in D$,

$$
\mathscr{L}_{D}\left(G_{D}(\cdot, \eta), x_{0}, r\right)=\min \left\{G_{D}\left(x_{0}, \eta\right), \tau(r)\right\}
$$

for every regular value of $r$.

Proof. Suppose first that $u \in C^{2}$. Let $s_{1}, s_{2}$ be regular values such that $r_{1}<s_{1}<s_{2}<r_{2}$, and put $A_{D}=A_{D}\left(x_{0}, s_{1}, s_{2}\right)$. For every $v \in C^{2}\left(A_{D}\right)$ we have, by Green's formula,

$$
\int_{A_{D}}(v L u-u L v) d x=\int_{\partial A_{D}}\langle A(v \nabla u-u \nabla v), \nu\rangle d \sigma .
$$

In particular, if $L v=0$ then

$$
\int_{A_{D}} v L u d x=\int_{\partial A_{D}}\langle A(v \nabla u-u \nabla v), \nu\rangle d \sigma
$$

Taking $v=1$, we obtain

$$
\int_{A_{D}} L u d x=\int_{\partial A_{D}}\langle A \nabla u, \nu\rangle d \sigma .
$$

If we put $v=G_{D}=G_{D}\left(x_{0}, \cdot\right)$ in (7), we obtain

$$
\int_{A_{D}} G_{D} L u d x=\tau\left(s_{2}\right) \kappa\left(s_{2}\right)-\tau\left(s_{1}\right) \kappa\left(s_{1}\right)+\mathscr{L}_{D}\left(s_{2}\right)-\mathscr{L}_{D}\left(s_{1}\right),
$$


where $\mathscr{L}_{D}(s)=\mathscr{L}_{D}\left(u, x_{0}, s\right)$ and

$$
\kappa(s)=\int_{\partial B_{D}\left(x_{0}, s\right)}\langle A \nabla u, \nu\rangle d \sigma .
$$

Note that, by (8),

$$
\kappa\left(s_{2}\right)-\kappa\left(s_{1}\right)=\int_{A_{D}} L u d x .
$$

It now follows, exactly as in the proof of Theorem 1, that

$$
\frac{\mathscr{L}_{D}(t)-\mathscr{L}_{D}(s)}{\tau(t)-\tau(s)} \leq \frac{\mathscr{L}_{D}(s)-\mathscr{L}_{D}(r)}{\tau(s)-\tau(r)}
$$

whenever $r, s, t$ are regular values such that $r_{1}<r<s<t<r_{2}$. Hence there is a convex function $\varphi$, defined on the $\tau$-image of the set of regular values in $] r_{1}, r_{2}[$, such that $\mathscr{L}_{D}=\varphi \circ \tau$. The discussion in Section 3 shows that $\varphi$ can be extended to a convex function on $] \tau\left(r_{2}\right), \tau\left(r_{1}\right)[$.

Now consider the general case. If $s_{1}, s_{2}$, and $A_{D}$ are as above, then by [10] there is a decreasing sequence $\left\{u_{j}\right\}$ of $C^{2}$ functions, such that $L u_{j} \geq 0$ for all $j$ and $u_{j} \rightarrow u$ on a neighbourhood of $\bar{A}_{D}$. For each $j$, there is a convex function $\varphi_{j}$ on $] \tau\left(s_{2}\right), \tau\left(s_{1}\right)\left[\right.$ such that $\mathscr{L}_{D}\left(u_{j}, x_{0}, r\right)=\varphi_{j}(\tau(r))$ for all regular values of $r$ in $] s_{1}, s_{2}[$. Hence

$$
\mathscr{L}_{D}\left(u, x_{0}, r\right)=\lim \varphi_{j}(\tau(r)),
$$

and $\lim \varphi_{j}$ is convex if it is finite at one point. Since $s_{1}, s_{2}$ are arbitrary, the result will follow from the finiteness of $\mathscr{L}_{D}$.

To prove the finiteness, we use a method based on that in [18] for the heat equation, and thus simultaneously establish (6). Let $\eta \in D$, and let $r$ be a regular value (corresponding to $x_{0}$ ). If $B=B_{D}\left(x_{0}, r\right)$, then $G_{B}\left(x_{0}, \cdot\right)=$ $G_{D}\left(x_{0}, \cdot\right)-\tau(r)$, so that it follows from a result in $[12$, p. 80] that the measure $-\left\langle A \nabla G_{D}\left(x_{0}, \cdot\right), \nu\right\rangle d \sigma$ on $\partial B$ is the $L$-harmonic measure relative to $B$ and $x_{0}$. Therefore $\mathscr{L}_{D}\left(G_{D}(\cdot, \eta), x_{0}, \cdot\right)$ is decreasing, and if $\eta \notin \bar{B}$ then $\mathscr{L}_{D}\left(G_{D}(\cdot, \eta), x_{0}, r\right)$ $=G_{D}\left(x_{0}, \eta\right)$ because $G_{D}(\cdot, \eta)$ is $L$-harmonic on an open superset of $\bar{B}$. Suppose that $\eta \in B$. Then $\eta \in B_{D}\left(x_{0}, t_{1}\right)$ for some regular value of $t_{1}<r$, so that, for any regular value of $t_{2}>r$, the function $G_{D}(\cdot, \eta)$ is $L$-harmonic on $A_{D}\left(x_{0}, t_{1}, t_{2}\right)$. Therefore, by the first part of this proof, there are constants $\lambda_{1}, \lambda_{2}$ such that

$$
\mathscr{L}_{D}\left(G_{D}(\cdot, \eta), x_{0}, t\right)=\lambda_{1} \tau(t)+\lambda_{2}
$$

for every regular value of $t$ in ] $t_{1}, t_{2}$ [. Since $t_{2}$ is arbitrary, this holds for every regular $t>t_{1}$. Because $G_{D}(\cdot, \eta)=G_{D}(\eta, \cdot)$, which has a continuous extension to zero on $\partial D$, for each $\varepsilon>0$ the set

$$
D_{\varepsilon}=\left\{x \in D: G_{D}(x, \eta) \geq \varepsilon\right\}
$$


is compact. Therefore, since $\left\{B_{D}\left(x_{0}, s\right): \tau(s)>0\right\}$ expands to $D$ as $s$ increases, we can choose $s$ such that $D_{\varepsilon} \subseteq B_{D}\left(x_{0}, s\right)$, so that for all regular values of $t>s$ we have

$$
\mathscr{L}_{D}\left(G_{D}(\cdot, \eta), x_{0}, t\right) \leq L_{D}\left(\varepsilon, x_{0}, t\right)=\varepsilon .
$$

It follows that $\lambda_{2}=0$, so that

$$
\mathscr{L}_{D}\left(G_{D}(\cdot, \eta), x_{0}, r\right)= \begin{cases}G_{D}\left(x_{0}, \eta\right) & \text { if } \eta \notin \bar{B}_{D}\left(x_{0}, r\right), \\ \lambda_{1} \tau(r) & \text { if } \eta \in B_{D}\left(x_{0}, r\right) .\end{cases}
$$

Since the mean is decreasing, $\lambda_{1} \leq 1$. Hence

$$
\mathscr{L}_{D}\left(G_{D}(\cdot, \eta), x_{0}, r\right) \leq \min \left\{G_{D}\left(x_{0}, \eta\right), \tau(r)\right\}
$$

for all regular values of $r$. If $s_{1}, s_{2}$ and $A_{D}$ are as above, there exist an $L$ potential $v$ on $D$ and an $L$-harmonic function $h$ on $A_{D}$ such that $u=h-v$ on $A_{D}$, by [1, p. 97]. Since the $L$-potentials on $D$ with point support are proportional $[8$, p. 563], there is a non-negative Radon measure $\mu$ on $D$ such that

$$
v(x)=\int_{D} G_{D}(x, \eta) d \mu(\eta)
$$

for all $x \in D$, by $\left[8\right.$, p. 481]. We can assume that $\mu$ is supported by $\bar{A}_{D}$, and hence is finite, because $\int_{D \backslash \bar{A}_{D}} G_{D}(\cdot, \eta) d \mu(\eta)$ is $L$-harmonic on $A_{D}$. Then, by Tonelli's theorem and (9)

$$
\mathscr{L}_{D}\left(v, x_{0}, r\right)=\int_{D} \mathscr{L}_{D}\left(G_{D}(\cdot, \eta), x_{0}, r\right) d \mu(y) \leq \tau(r) \mu(D)<\infty
$$

for every regular value of $r$ in $] s_{1}, s_{2}\left[\right.$. Since $\mathscr{L}_{D}\left(h, x_{0}, \cdot\right)$ is obviously finite-valued, the convexity of $\mathscr{L}_{D}\left(u, x_{0}, \cdot\right)$ follows.

Finally, $\mathscr{L}_{D}\left(G_{D}(\cdot, \eta), x_{0}, \cdot\right)$ is the restriction of a function continuous on the set $\{r: \tau(r)>0\}$, so that equality holds in (9), and (6) is established. p. 6].

Remarks. (i) For the case $L=\Delta, D=\mathbf{R}^{n}$, the identity (6) is given in [3,

(ii) It is easy to show that an $L$-subharmonic function $u$ has an $L$-harmonic majorant on $D$ if and only if $\mathscr{L}_{D}\left(u, x_{0}, \cdot\right)$ is bounded above. The case $L=\Delta$ is given in [19].

(iii) The corresponding volume means can be developed and their properties established, exactly as in [19].

When Dinghas gave his proof of Theorem 1, he also proved similar results for the corresponding $L^{p}$ means of non-negative subharmonic functions [2]. These were extended to means over level surfaces of Green functions in [19], by an essentially similar method. We now prove analogues in the present setting, using the method of Fugard [4] which, conveniently, requires only that the surfaces used are level surfaces of some $L$-harmonic function. Since [4] is not readily accessible, we include the details. 
Theorem 3. If $u$ is $L$-subharmonic on $A_{D}\left(x_{0}, r_{1}, r_{2}\right)$, then there are convex functions $\varphi_{p}, 1<p \leq \infty$, on $] \tau\left(r_{2}\right), \tau\left(r_{1}\right)[$ such that

$$
\begin{aligned}
\mathscr{L}_{D}\left(\left(u^{+}\right)^{p}, x_{0}, r\right)^{1 / p} & =\varphi_{p}(\tau(r)) \quad(1<p<\infty), \\
\log \mathscr{L}_{D}\left(e^{u}, x_{0}, r\right) & =\varphi_{\infty}(\tau(r))
\end{aligned}
$$

for all regular values of $r$ in $] r_{1}, r_{2}[$.

Proof. Theorem 1 (ii) in [6] implies that $\left(u^{+}\right)^{p}$ and $e^{u}$ are $L$-subharmonic on $A_{D}\left(x_{0}, r_{1}, r_{2}\right)$, so that the finiteness follows from Theorem 2 .

Consider the case $1<p<\infty$. We can obviously suppose that $u \geq 0$, and by considering $u+k^{-1}$ and then making $k \rightarrow \infty$, we can further suppose that $u>0$. We shall omit the subscript $D$, and put

$$
\mathscr{L}_{p}(u, r)=\mathscr{L}\left(u^{p}, x_{0}, r\right)^{1 / p}
$$

Let $r, s, t$ be regular values such that $r_{1}<r<s<t<r_{2}$. In view of the discussion in Section 3, we have only to establish that

$$
\mathscr{L}_{p}(u, s) \leq\left(\frac{\tau(t)-\tau(s)}{\tau(t)-\tau(r)}\right) \mathscr{L}_{p}(u, r)+\left(\frac{\tau(s)-\tau(r)}{\tau(t)-\tau(r)}\right) \mathscr{L}_{p}(u, t)
$$

If $\mathscr{L}_{p}(u, r)=\mathscr{L}_{p}(u, t)$, then $(10)$ reduces to $\mathscr{L}_{p}(u, s) \leq \mathscr{L}_{p}(u, t)$, which follows from Theorem 2 applied to $u^{p}$. Suppose that $\mathscr{L}_{p}(u, t)<\mathscr{L}_{p}(u, r)$; the case $\mathscr{L}_{p}(u, r)<\mathscr{L}_{p}(u, t)$ is similar. For any number $a<\tau(t)$, the function $G_{D}\left(x_{0}, \cdot\right)-a$ is positive and $L$-superharmonic on an open superset of $\bar{A}_{D}\left(x_{0}, r, t\right)$, so that the function

$$
v=\left(G_{D}\left(x_{0}, \cdot\right)-a\right)^{1-p} u^{p}
$$

is $L$-subharmonic on such a set, by Theorem 1 (iii) in [6]. Therefore, by Theorem 2 applied to $v$,

$$
\begin{aligned}
(\tau(s)-a)^{1-p} \mathscr{L}\left(u^{p}, s\right) \leq & \left(\frac{\tau(t)-\tau(s)}{\tau(t)-\tau(r)}\right)(\tau(r)-a)^{1-p} \mathscr{L}\left(u^{p}, r\right) \\
& +\left(\frac{\tau(s)-\tau(r)}{\tau(t)-\tau(r)}\right)(\tau(t)-a)^{1-p} \mathscr{L}\left(u^{p}, t\right) .
\end{aligned}
$$

If we take

$$
a=\frac{\tau(r) \mathscr{L}_{p}(u, t)-\tau(t) \mathscr{L}_{p}(u, r)}{\mathscr{L}_{p}(u, t)-\mathscr{L}_{p}(u, r)}
$$


and calculate $\tau(s)-a, \tau(r)-a$, and $\tau(t)-a$, then (11) yields

$$
\begin{aligned}
& \left((\tau(s)-\tau(r)) \mathscr{L}_{p}(u, t)+(\tau(t)-\tau(s)) \mathscr{L}_{p}(u, r)\right)^{1-p} \mathscr{L}\left(u^{p}, s\right) \\
& \leq \frac{(\tau(t)-\tau(s))}{(\tau(t)-\tau(r))^{p}} \mathscr{L}_{p}(u, r)^{1-p} \mathscr{L}\left(u^{p}, r\right)+\frac{(\tau(s)-\tau(r))}{(\tau(t)-\tau(r))^{p}} \mathscr{L}_{p}(u, t)^{1-p} \mathscr{L}\left(u^{p}, t\right) \\
& =\frac{(\tau(t)-\tau(s)) \mathscr{L}_{p}(u, r)+(\tau(s)-\tau(r)) \mathscr{L}_{p}(u, t)}{(\tau(t)-\tau(r))^{p}}
\end{aligned}
$$

which easily reduces to (10).

Now consider the case $p=\infty$, again omitting the subscripts $D$, and taking $r, s, t$ as before. We need consider only the case $\mathscr{L}\left(e^{u}, t\right)<\mathscr{L}\left(e^{u}, r\right)$. Here

$$
b=\frac{\log \mathscr{L}\left(e^{u}, t\right)-\log \mathscr{L}\left(e^{u}, r\right)}{\tau(r)-\tau(t)}<0
$$

so that the function $w=u+b G_{D}\left(x_{0}, \cdot\right)$ is $L$-subharmonic on an open superset of $\bar{A}_{D}\left(x_{0}, r, t\right)$. Therefore, by Theorem 2 applied to $e^{w}$,

$$
e^{b \tau(s)} \mathscr{L}\left(e^{u}, s\right) \leq\left(\frac{\tau(t)-\tau(s)}{\tau(t)-\tau(r)}\right) e^{b \tau(r)} \mathscr{L}\left(e^{u}, r\right)+\left(\frac{\tau(s)-\tau(r)}{\tau(t)-\tau(r)}\right) e^{b \tau(t)} \mathscr{L}\left(e^{u}, t\right) .
$$

Calculating $b \tau(\varrho)+\log \mathscr{L}\left(e^{u}, \varrho\right)$ for all $\varrho \in\{r, s, t\}$, we first see that

$$
b \tau(r)+\log \mathscr{L}\left(e^{u}, r\right)=b \tau(t)+\log \mathscr{L}\left(e^{u}, t\right)
$$

so that

$$
e^{b \tau(s)} \mathscr{L}\left(e^{u}, s\right) \leq e^{b \tau(r)} \log \mathscr{L}\left(e^{u}, r\right)
$$

and then that

$$
\begin{gathered}
\tau(s) \log \mathscr{L}\left(e^{u}, t\right)-\tau(s) \log \mathscr{L}\left(e^{u}, r\right)+(\tau(r)-\tau(t)) \log \mathscr{L}\left(e^{u}, s\right) \\
\leq \tau(r) \log \mathscr{L}\left(e^{u}, t\right)-\tau(t) \log \mathscr{L}\left(e^{u}, r\right),
\end{gathered}
$$

which is easily rearranged to the desired inequality, and the result follows as before. 


\section{The heat equation}

Let $\theta$ denote the heat operator $\sum_{i=1}^{n} D_{i}^{2}-D_{t}$, and $\theta^{*}$ its adjoint, in $\mathbf{R}^{n+1}=$ $\left\{p=(x, t): x \in \mathbf{R}^{n}, t \in \mathbf{R}\right\}$. All concepts relative to the adjoint will carry the prefix $\theta^{*}$. A typical point of $\mathbf{R}^{n+1}$ will be denoted by $p$ or $(x, t)(q$ or $(y, s)$, etc.), whichever is convenient. We put

$$
\nabla_{x} u=\left(D_{1} u, \ldots, D_{n} u\right), \quad \nabla u=\left(\nabla_{x} u, D_{t} u\right), \quad\langle x, y\rangle=\sum_{i=1}^{n} x_{i} y_{i}
$$

and use $\|\cdot\|$ to denote the Euclidean norm in both $\mathbf{R}^{n}$ and $\mathbf{R}^{n+1}$. Integrals with respect to $(n+1)$-dimensional Lebesgue measure are denoted by $\int d p$, and those with respect to $n$-dimensional Lebesgue measure by $\int d x$. A temperature is a solution of the heat equation, and subtemperatures and supertemperatures are the corresponding subsolutions and supersolutions $[3,16,17]$.

Let $D$ be an open subset of $\mathbf{R}^{n+1}$ which is $\theta^{*}$-Dirichlet regular, and let $G_{D}$ denote its Green function (in the sense of [17]), except that the subscript is omitted if $D=\mathbf{R}^{n+1}$. If $p_{0} \in D$, then $G_{D}\left(p_{0}, \cdot\right)$ is a non-negative $\theta^{*}$-supertemperature on $D$, and a $\theta^{*}$-temperature on $D \backslash\left\{p_{0}\right\}$. Furthermore, there is a positive, bounded, $\theta^{*}$-temperature $h$ on $D$ such that

$$
G_{D}\left(p_{0}, \cdot\right)=G\left(p_{0}, \cdot\right)-h
$$

so that $G_{D}\left(p_{0}, \cdot\right) \in C^{\infty}\left(D \backslash\left\{p_{0}\right\}\right)$. (The function $h$ is the PWB solution of the $\theta^{*}$-Dirichlet problem on $D$ with boundary values $G\left(p_{0}, \cdot\right)[17 ; 3$, p. 331].) It therefore follows from Sard's theorem [15, p. 45] that, for almost every $c>0$, the set

$$
\left\{p \in D: G_{D}\left(p_{0}, p\right)=(4 \pi c)^{-n / 2}\right\}
$$

is a $C^{\infty}$, regular, $n$-dimensional manifold. We call such a value of $c$ a regular value. For an arbitrary positive value of $c$, we put

$$
\Omega_{D}\left(p_{0}, c\right)=\left\{p \in D: G_{D}\left(p_{0}, p\right)>(4 \pi c)^{-n / 2}\right\} .
$$

For any regular value of $c$, the union of $\left\{p_{0}\right\}$ with the set $(13)$ is $\partial \Omega_{D}\left(p_{0}, c\right)$. Since $G_{D}\left(p_{0}, \cdot\right)$ is lower semicontinuous, any $\Omega_{D}\left(p_{0}, c\right)$ is open. The assumption that $D$ is $\theta^{*}$-Dirichlet regular implies that $G_{D}\left(p_{0}, \cdot\right)$ can be continuously extended to zero on $\partial D$, so that $\bar{\Omega}_{D}\left(p_{0}, c\right)$ is a compact subset of $D$. In view of (12), there is a positive constant $\delta$ such that $G\left(p_{0}, \cdot\right)-\delta \leq G_{D}\left(p_{0}, \cdot\right) \leq G\left(p_{0}, \cdot\right)$ on $D$, so that if $d$ satisfies $(4 \pi d)^{-n / 2}=(4 \pi c)^{-n / 2}+\delta$, we have

$$
\Omega\left(p_{0}, d\right) \leq \Omega_{D}\left(p_{0}, c\right) \subseteq \Omega\left(p_{0}, c\right) .
$$


Furthermore, $\Omega_{D}\left(p_{0}, c\right)$ is connected; see [20] for details. If $c$ is a regular value, then the outward unit normal $\nu=\left(\nu_{x}, \nu_{t}\right)$ to $\partial \Omega_{D}\left(p_{0}, c\right)$ is given by

$$
\nu=-\nabla G_{D}\left(p_{0}, \cdot\right)\left\|\nabla G_{D}\left(p_{0}, \cdot\right)\right\|^{-1}
$$

and we put

$$
\mathscr{M}_{D}\left(u, p_{0}, c\right)=\int_{\partial \Omega_{D}\left(p_{0}, c\right)} K_{D}\left(p_{0}, \cdot\right) u d \sigma
$$

whenever the integral exists, where

$$
K_{D}\left(p_{0}, \cdot\right)=\left\|\nabla_{x} G_{D}\left(p_{0}, \cdot\right)\right\|^{2}\left\|\nabla G_{D}\left(p_{0}, \cdot\right)\right\|^{-1}=-\left\langle\nabla_{x} G_{D}\left(p_{0}, \cdot\right), \nu_{x}\right\rangle
$$

on $\partial \Omega_{D}\left(p_{0}, c\right) \backslash\left\{p_{0}\right\}$, and $\sigma$ denotes the surface area measure. It was proved in [20] that $K_{D}\left(p_{0}, \cdot\right)$ is bounded on $\partial \Omega_{D}\left(p_{0}, c\right) \backslash\left\{p_{0}\right\}$.

Finally, if $c_{1}, c_{2}$ are regular values with $c_{1}<c_{2}$, we put

$$
A_{D}\left(p_{0}, c_{1}, c_{2}\right)=\Omega_{D}\left(p_{0}, c_{2}\right) \backslash \bar{\Omega}_{D}\left(p_{0}, c_{1}\right) .
$$

We can now give a simpler proof of [20, Theorem 3]. In the case $D=\mathbf{R}^{n+1}$, the finiteness of the means was proved in [18].

Theorem 4. Let $u$ be a subtemperature on an open superset $E$ of $\bar{A}_{D}\left(p_{0}, c_{1}, c_{2}\right)$. Then there is a function $\varphi$, either finite and convex or identically $-\infty$, such that $\mathscr{M}_{D}\left(u, p_{0}, c\right)=\varphi\left(c^{-n / 2}\right)$ for all regular values of $c$ in $\left[c_{1}, c_{2}\right]$.

Proof. Suppose first that $u \in C^{2}$. Let $U$ by any bounded domain whose boundary is smooth enough for the divergence theorem to be applicable, and whose closure lies in $E$. It follows from Green's formula for the heat equation that, whenever $v$ is a $\theta^{*}$-temperature on a neighbourhood of $\bar{U}$, we have

$$
\int_{U} v \theta u d p=\int_{\partial U}\left(\left\langle v \nabla_{x} u-u \nabla_{x} v, \nu_{x}\right\rangle-u v \nu_{t}\right) d \sigma
$$

where $\left(\nu_{x}, \nu_{t}\right)$ is the outward unit normal to $\partial U$. Taking $v=1$ we obtain

$$
\int_{U} \theta u d p=\int_{\partial U}\left(\left\langle\nabla_{x} u, \nu_{x}\right\rangle-u \nu_{t}\right) d \sigma
$$

Let $d_{1}, d_{2}$ be regular values such that $c_{1} \leq d_{1}<d_{2} \leq c_{2}$, and put $A_{D}=$ $A_{D}\left(p_{0}, d_{1}, d_{2}\right)$. We would like to use (15) with $U=A_{D}$ and $v=G_{D}\left(p_{0}, \cdot\right)$, but the smoothness of this choice of $v$ breaks down at $p_{0}$. We must therefore employ an approximation argument. For $t<t_{0}$, put

$$
F\left(d_{i}, t\right)=\partial \Omega_{D}\left(p_{0}, d_{i}\right) \cap\left(\mathbf{R}^{n} \times\right]-\infty, t[) \quad \text { for } i \in\{1,2\},
$$




$$
V(t)=A_{D} \cap\left(\mathbf{R}^{n} \times\right]-\infty, t[) \quad \text { and } \quad T(t)=A_{D} \cap\left(\mathbf{R}^{n} \times\{t\}\right),
$$

provided that the intersections are not empty. Applying (15) to the components of $V(t)$, with $v=G_{D}=G_{D}\left(p_{0}, \cdot\right)$, we obtain

$$
\int_{V(t)} G_{D} \theta u d p=\int_{\partial V(t)}\left(\left\langle G_{D} \nabla_{x} u-u \nabla_{x} G_{D}, \nu_{x}\right\rangle-u G_{D} \nu_{t}\right) d \sigma .
$$

On $T(t)$ we have $\nu_{x}=0, \nu_{t}=1$ and $d \sigma=d x$, so that

$$
\int_{T(t)}\left(\left\langle G_{D} \nabla_{x} u-u \nabla_{x} G_{D}, \nu_{x}\right\rangle-u G_{D} \nu_{t}\right) d \sigma=\int_{T(t)}-u G_{D} d x
$$

which tends to zero as $t \rightarrow t_{0}$-, because the integrand is bounded on $A_{D}$ and the Lebesgue measure of $T(t)$ tends to zero (since $A_{D} \subseteq \Omega_{D}\left(p_{0}, d_{2}\right) \subseteq \Omega\left(p_{0}, d_{2}\right)$ by (14)). Next, as $K_{D}\left(p_{0}, \cdot\right)$ is bounded on $\partial \Omega\left(p_{0}, d_{i}\right)$ for each $i$, and (14) holds,

$$
\int_{\partial V(t) \backslash T(t)}\left\langle-u \nabla_{x} G_{D}, \nu_{x}\right\rangle d \sigma \rightarrow \mathscr{M}_{D}\left(u, p_{0}, d_{2}\right)-\mathscr{M}_{D}\left(u, p_{0}, d_{1}\right)
$$

as $t \rightarrow t_{0}-$. Also, since $G_{D}=\left(4 \pi d_{i}\right)^{-n / 2}$ on $\partial \Omega_{D}\left(p_{0}, d_{i}\right)$, as $t \rightarrow t_{0}-$ we have

$$
\begin{aligned}
\int_{\partial V(t) \backslash T(t)} & \left(\left\langle G_{D} \nabla_{x} u, \nu_{x}\right\rangle-u G_{D} \nu_{t}\right) d \sigma \\
\rightarrow & \left(4 \pi d_{2}\right)^{-n / 2} \int_{\partial \Omega_{D}\left(p_{0}, d_{2}\right)}\left(\left\langle\nabla_{x} u, \nu_{x}\right\rangle-u \nu_{t}\right) d \sigma \\
& \quad-\left(4 \pi d_{1}\right)^{-n / 2} \int_{\partial \Omega_{D}\left(p_{0}, d_{1}\right)}\left(\left\langle\nabla_{x} u, \nu_{x}\right\rangle-u \nu_{t}\right) d \sigma .
\end{aligned}
$$

Making $t \rightarrow t_{0}-$ in (17), we thus obtain

$$
\int_{A_{D}} G_{D} \theta u d p=\mathscr{M}_{D}\left(u, p_{0}, d_{2}\right)-\mathscr{M}_{D}\left(u, p_{0}, d_{1}\right)+\left(4 \pi d_{2}\right)^{-n / 2} \lambda\left(d_{2}\right)-\left(4 \pi d_{1}\right)^{-n / 2} \lambda\left(d_{1}\right),
$$

where

$$
\lambda(c)=\int_{\partial \Omega_{D}\left(p_{0}, c\right)}\left(\left\langle\nabla_{x} u, \nu_{x}\right\rangle-u \nu_{t}\right) d \sigma
$$

and, by (16) with $U=A_{D}$,

$$
\lambda\left(d_{2}\right)-\lambda\left(d_{1}\right)=\int_{A_{D}} \theta u d p .
$$

The result now follows exactly as for the subharmonic case; the necessary approximation theorem is given in [3, p. 281]. 


\section{References}

[1] Brelot, M.: Lectures on potential theory. - Tata Institute of Fundamental Research, Bombay, 1960.

[2] Dinghas, A.: Über einige Konvexitätsätze für die Mittelwerte von subharmonischen Funktionen. - J. Math. Pures Appl. 44, 1965, 223-247.

[3] Dоов, J.L.: Classical potential theory and its probabilistic counterpart. - Springer-Verlag, New York, 1984.

[4] FugARD, T.B.: Growth and convexity properties of harmonic and subharmonic functions. - M. Sc. Thesis, The Queen's University of Belfast, 1979.

[5] Gardiner, S.J.: Subharmonicity of mean values of subharmonic functions. - Proc. Roy. Irish Acad. 87 A, 1987, 169-175.

[6] Gardiner, S.J., and M. Klimek: Convexity and subsolutions of partial differential equations. - Bull. London Math. Soc. 18, 1986, 41-43.

[7] Goldberg, R.R.: Methods of real analysis, 2nd edition. - Wiley \& Sons, New York, 1976.

[8] HERVÉ, R.M.: Recherches axiomatiques sur la théorie des fonctions surharmoniques et du potentiel. - Ann. Inst. Fourier 12, 1962, 415-571.

[9] Нон, W., and N. ЈАсов: Remarks on mean value properties of solutions of second order differential operators. - Exposit. Math. 9, 1991, 367-377.

[10] Littman, W.: Generalized subharmonic functions: Monotonic approximations and an improved maximum principle. - Ann. Scuola Norm. Sup. Pisa Cl. Sci. (4) 17, 1963, 207-222.

[11] Littman, W., G. Stampacchia, and H. Weinberger: Regular points for elliptic equations with discontinuous coefficients. - Ann. Scuola Norm. Sup. Pisa Cl. Sci. (4) 17, 1963, 43-77.

[12] Miranda, C.: Partial differential equations of elliptic type, 2nd edition. - Springer-Verlag, Berlin, 1970.

[13] RiEsz, F.: Sur les fonctions subharmoniques et leur rapport à la théorie du potentiel. I. Acta Math. 48, 1926, 329-343.

[14] Sternberg, S.: Lectures on differential geometry. - Prentice-Hall, Englewood Cliffs, 1964.

[15] Stromberg, K.R.: An introduction to classical real analysis. - Wadsworth, Belmont, 1981.

[16] Watson, N.A.: A theory of subtemperatures in several variables. - Proc. London Math. Soc. (3) 26, 1973, 385-417.

[17] Watson, N.A.: Green functions, potentials, and the Dirichlet problem for the heat equation. - Proc. London Math. Soc. (3) 33, 1976, 251-298; Corrigendum, ibid. (3) 37, $1978,32-34$.

[18] Watson, N.A.: A convexity theorem for local mean values of subtemperatures. - Bull. London Math. Soc. 22, 1990, 245-252.

[19] Watson, N.A.: Mean values of subharmonic functions over Green spheres. - Math. Scand. (to appear).

[20] Watson, N.A.: Mean values of subtemperatures over level surfaces of Green functions. Ark. Mat. (to appear). 